\title{
Oberflächendiffusion von Wolfram
}

\author{
G. M. Neumann, W. Hirschwald und I. N. Stranski \\ Institut für Physikalische Chemie der Freien Universität Berlin \\ und Fritz-Haber-Institut der Max-Planck-Gesellschaft, Berlin-Dahlem
}

(Z. Naturforschg. 21 a, 807-811 [1966]; eingegangen am 25. Februar 1966)

\begin{abstract}
Es wurde die Oberflächenwanderung von Wolfram auf Wolfram mit Hilfe des radioaktiven Isotops Wolfram-185 untersucht. Für den Temperaturbereich 1700 bis $2300^{\circ} \mathrm{C}$ wurde für den Koeffizienten der Oberflächendiffusion die Beziehung $D=1,1 \cdot 10^{-3} \exp \{-31600 /(R T)\}$ gefunden. Die gemessene Platzwechselenergie entspricht dem nach der Methode von Stranski und Sunrmann abgeschätzten Wert für den Übergang in der Fläche. Gleichartige Berechnungen für eine Reihe von Metallen zeigen eine sehr gute Übereinstimmung der berechneten Aktivierungsenergie mit experimentellen Daten aus der Literatur.
\end{abstract}

Die Untersuchungen über die Oberflächendiffusion von Metallatomen auf dem eigenen Kristallgitter haben in den letzten Jahren immer mehr Interesse gefunden. Auf der einen Seite stehen Untersuchungen mit dem Feldelektronen- oder dem Feldionenmikroskop, auf der anderen Seite Untersuchungen nach der "masstransfer"-Technik oder der radioaktiven Markierungstechnik.

Untersuchungen der Oberflächendiffusion von Wolfram auf Wolfram mit dem Feldelektronenmikroskop liegen bereits in einer Reihe von Arbeiten ${ }^{1-7}$ vor; Untersuchungen nach einer der beiden anderen Methoden sind hierüber bis jetzt noch nicht bekannt geworden. In dieser Arbeit wird daher über die Wanderung von radioaktiven Wolframatomen auf Wolfram berichtet.

\section{Experimenteller Teil}

Als Trägermaterial diente Wolframband $(99,9 \% \mathrm{~W}$, Spuren Mo, Al, Mn, Si, Fe - Länge 100 mm, Breite $1 \mathrm{~mm}$, Stärke 0,1 mm), das von der Firma O s r a m $\mathrm{GmbH}$ Berlin bezogen wurde.

Für die Untersuchung der Oberflächendiffusion wurde ein $0,2 \mathrm{~mm}$ breiter Streifen in der Mitte des Wolframbandes mit radioaktivem Ammoniumparawolframat indiziert, das im Wasserstoffstrom bei $1200^{\circ} \mathrm{C}$ zu metallischem Wolfram reduziert wurde. Das radioaktive Wolframisotop W-185 wurde in Form von Wolframtrioxid aus Harwell/England bezogen.

Die Versuchsanordnung ist aus Abb. 1 zu ersehen. In ein zylindrisches Rohr aus Thüringer Glas wurden

1 E. W. Müller, Z. Phys. 126, 642 [1949].

2 I. L. SокоцSкAJA, Izv. Akad. Nauk SSSR 20, 1151 [1956]; Soviet Phys. - Techn. Phys. 1, 1147 [1957].

3 P. C. Bettler u. F. M. Charbonnier, Phys. Rev. 119, 85 [1960].

4 J.P. Barbour, F. M. Charbonnier, W.W. Dolan, W. P. Dyke, E. E. Martin u. J. K. Trolan, Phys. Rev. 117, 1452 [1960]. zwei Fe-Ni-Stäbe (Vacovit 501 der Vacuumschmelze AG Hanau) von 2,5 mm Durchmesser und zwei Fe-NiDrähte von $1 \mathrm{~mm}$ Durchmesser eingeschmolzen. An ihren Enden wurde mit Lüsterklemmen die eigentliche Haltevorrichtung für das Wolframband angeschraubt. Die Fe-Ni-Stäbe dienten als Stromzuführung, die Drähte zur Spannungsmessung. Die Konstruktion der Haltevorrichtung für das Wolframband ist aus Abb.l ersichtlich. Das Wolframband wurde zwischen den Fe-NiDrähten durch Punktschweißung befestigt.

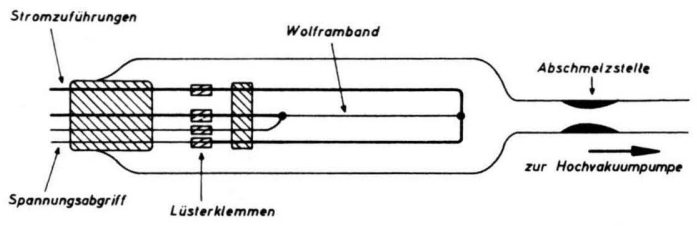

Abb. 1. Versuchsapparatur.

Die Aktivitätsverteilung auf dem Wolframband wurde mit einem Endfensterzählrohr FHZ 12 in Verbindung mit dem Strahlungsmeßgerät FH 49 der Firma F ri e s e k e \& H o e p f n e r, Erlangen, ermittelt. Das Zählrohr wurde zur Abschirmung in eine Bleikammer gebracht, die unten mit einer $5 \mathrm{~mm}$ starken Al-Platte abgedeckt war. In der Mitte der Al-Platte war dem Glimmerfenster des Zählrohrs gegenüber ein Schlitz von $0,2 \mathrm{~mm}$ Breite angebracht, so daß die vom Wolframband emittierte Strahlung im wesentlichen nur durch diesen Spalt in das Zählrohr eintreten konnte. Zur Aktivitätsmessung wurde das Wolframband mit der Haltevorrichtung unterhalb der Bleikammer am Schlitz vorbeigeführt und die Zählrate von Millimeter zu Millimeter über eine Zeitdauer von je 10 Minuten bestimmt. Die gemessenen Zählraten wurden in Hinblick auf

5 Y. Matsuo, Wakayama Daigaku Gakugeigakubu Kiyo, Shizen Kagaku 12, 61 [1962].

${ }^{6}$ G. Ehrlich u. F. G. Hudda, 3rd Intern. Congr. on Catalysis, Amsterdam 1964.

7 D. W. Basset, Proc. Roy. Soc. London A 286, 191 [1965]. 
Nulleffekt und Zerfallszeit korrigiert, eine Korrektur für die Totzeit der Zählanordnung konnte in Anbetracht der geringen Zählraten unterbleiben. Ferner wurde die Meßanordnung vor und nach jeder Meßreihe auf Konstanz der Meßbedingungen geprüft. Die Temperatur des Wolframbandes wurde pyrometrisch mit einem Mikropyrometer der Py ro-W e rk e GmbH, Hannover, sowie aus dem elektrischen Widerstand über eine Strom-Spannungsmessung ermittelt. Die Temperaturkonstanz wurde dabei durch Konstanthaltung des Produktes $U \cdot I^{1 / 3}$ erreicht.

Nach der Ermittlung der Anfangsverteilung wurde das Wolframband mit der Haltevorrichtung montiert, der Versuchskolben angeschmolzen und die Apparatur evakuiert. Nach 12- bis 15-stündigem Ausheizen bei $400{ }^{\circ} \mathrm{C}$ wurde der Versuchskolben bei laufender Pumpe abgeschmolzen und die Diffusionsglühung durch direktes Erhitzen des Wolframbandes auf Temperaturen von 1700 bis $2300{ }^{\circ} \mathrm{C}$ bei Versuchszeiten von 70 bis 230 Stunden durchgeführt. Der Druck während der Diffusionsversuche lag stets im Bereich von $10^{-7}$ Torr, wie mit einem angeschmolzenen Alpert-Ionisationsmanometer festgestellt wurde. Nach Beendigung des Versuches wurde die Apparatur aufgesprengt und die Endverteilung bestimmt.

\section{Ergebnisse}

Zur Auswertung der Meßergebnisse wurden in einem Diagramm die gemessenen Zählraten gegen die jeweiligen Entfernungen vom Nullpunkt der Verteilung, d. h. dem Aktivitätsmaximum aufgetragen. Zur rechnerischen Auswertung der Meßergebnisse läßt sich bei den vorliegenden Versuchsbedingungen eine aus dem Quellenintegral abgeleitete Lösung des zweiten Fickschen Gesetzes anwenden.

$$
N=\frac{N_{0}}{2 \sqrt{\pi D t}} \exp \left\{-x^{2} /(4 D t)\right\} ;
$$

dabei ist $N=$ Aktivität an der Stelle $x, N_{0}=$ Aktivität an der Stelle $x=0, x=$ Entfernung vom Nullpunkt der Verteilung, $D=$ Diffusionskoeffizient, $t=$ Diffusionsdauer.

Der Diffusionskoeffizient läßt sich dann aus dem Anstieg der im $\log N / x^{2}$-Diagramm erhaltenen Geraden durch Einsetzen der Werte für den Diffusionsweg und die Diffusionsdauer berechnen:

$\log N=-0,4343 \cdot x^{2} /(4 D t)+\log \left\{N_{0} /(2 \sqrt{\pi D t})\right\}$.

Die Gültigkeit dieser Lösung nicht nur für Quellen linienförmiger Ausdehnung, sondern auch für Quellen durchaus endlicher Breite haben Berechnungen von Harrison und Andelin ${ }^{8}$ ergeben, so daß eine

8 B. K. Harrison u. R. L. Andelin, USAEC-LA 2880 [1963].
Anwendung dieser Lösung auf die vorliegenden Grenzbedingungen gerechtfertigt erscheint.

Die Auswertung der Meßergebnisse ergab die in Tab. 1 zusammengefaßten Diffusionskoeffizienten.

\begin{tabular}{|c|c|c|c|}
\hline \multicolumn{2}{|c|}{ Temperatur } & $\begin{array}{c}\text { Versuchs- } \\
\text { dauer } \\
\mathrm{h}\end{array}$ & $\begin{array}{c}\text { Diffusions- } \\
\text { koeffizient } \\
\mathrm{cm}^{2} / \mathrm{sec}\end{array}$ \\
\hline${ }^{\circ} \mathrm{K}$ & ${ }^{\circ} \mathrm{K}^{-1}$ & & $3,62 \cdot 10^{-7}$ \\
2002 & $4,99 \cdot 10^{-4}$ & 234 & $4,59 \cdot 10^{-7}$ \\
2054 & $4,87 \cdot 10^{-4}$ & 100 & $5,58 \cdot 10^{-7}$ \\
2106 & $4,74 \cdot 10^{-4}$ & 98,5 & $6,32 \cdot 10^{-7}$ \\
2156 & $4,64 \cdot 10^{-4}$ & 168 & $8,54 \cdot 10^{-7}$ \\
2210 & $4,53 \cdot 10^{-4}$ & 144 & $9,23 \cdot 10^{-7}$ \\
2260 & $4,43 \cdot 10^{-4}$ & 100 & $1,22 \cdot 10^{-6}$ \\
2316 & $4,33 \cdot 10^{-4}$ & 98,5 & $1,35 \cdot 10^{-6}$ \\
2370 & $4,22 \cdot 10^{-4}$ & 96 & $1,48 \cdot 10^{-6}$ \\
2422 & $4,13 \cdot 10^{-4}$ & 105 & $1,83 \cdot 10^{-6}$ \\
2475 & $4,04 \cdot 10^{-4}$ & 96 & $1,97 \cdot 10^{-6}$ \\
2526 & $3,96 \cdot 10^{-4}$ & 72 & \\
\hline
\end{tabular}

Tab. 1. Oberflächendiffusionskoeffizienten von Wolfram.

Die Temperaturabhängigkeit des Diffusionskoeffizienten folgt der Arrheniusschen Gleichung und läßt sich beschreiben durch die Beziehung:

$$
D=1,1 \cdot 10^{-3} \exp \{-31600 /(R T)\} .
$$

Der größte Fehler bei den Messungen ist bedingt durch den mittleren statistischen Fehler der Aktivitätsbestimmung, der etwa $10 \%$ beträgt. Dazu kommt eine Ungenauigkeit in der Temperaturbestimmung von etwa $2 \%$ sowie ein Fehler infolge der endlichen Ausdehnung der Quelle von ewa 8\%. Damit ergibt sich ein Gesamtfehler von etwa $20 \%$.

\section{Diskussion}

Die experimentell ermittelte Aktivierungsenergie eröffnet eine Möglichkeit, den Mechanismus der Oberflächenwanderung auf Grund molekularkinetischer Betrachtungen der Einzelvorgänge an der Kristalloberfläche zu deuten.

Der Idealkristall stellt ein aus einzelnen atomaren Bausteinen regelmäßig aufgebautes Gitter dar, dessen Oberflächenbausteine sich ebenfalls auf normalen Gitterpunkten befinden, wobei diese nach Kossel und Stranski je nach ihrer Lage verschieden stark gebunden sind. In der Abb. 3 sind die hauptsächlich in Betracht kommenden Lagen einzelner Oberflächenbausteine dargestellt. Für die Bausteine über einer (110)-Fläche stehen dabei zwei verschiedene Lagen zur Verfügung - der normale Gitterplatz (a) in der nächsthöheren Netzebene mit den Nachbarzahlen 


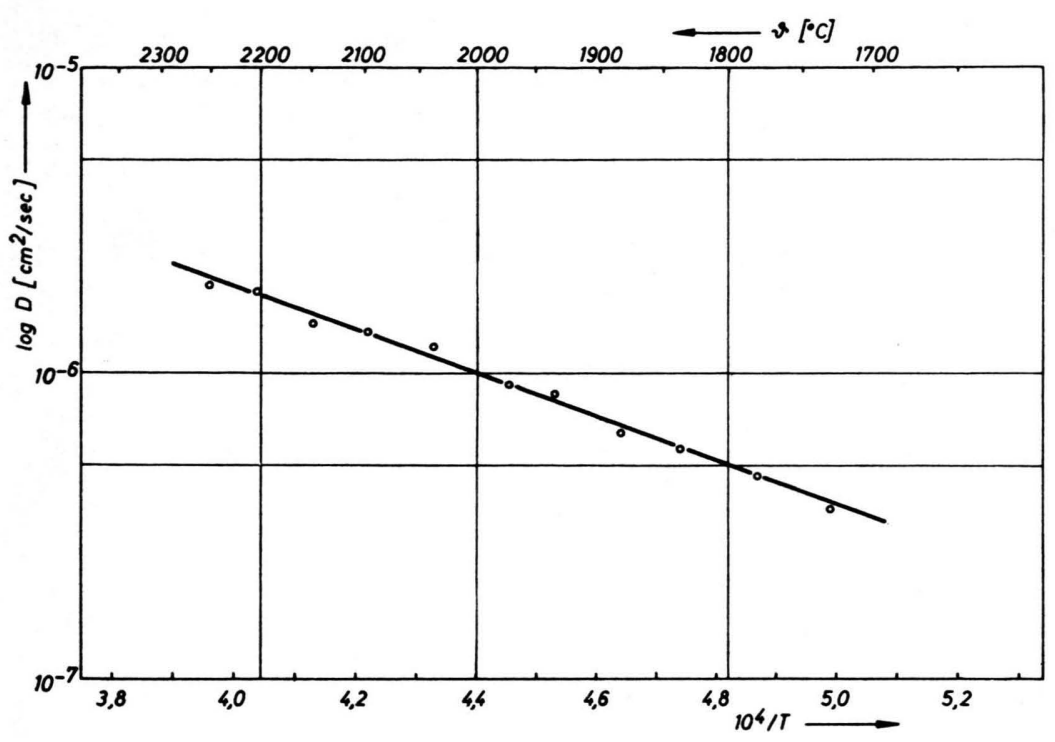

Abb. 2. Temperaturabhängigkeit des Oberflächendiffusionskoeffizienten von Wolfram.

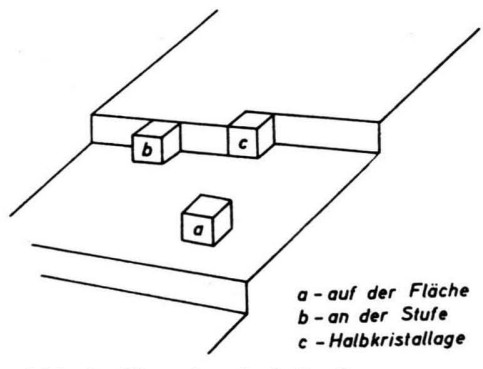

Abb. 3. Charakteristische Lagen von Oberflächenbausteinen.
2/2/5 und eine energetisch tiefere Lage $\left(a_{1}\right)$ mit den Nachbarzahlen $3 / 1 / 2$, d. h. in unmittelbarer Berührung mit drei Nachbarbausteinen (Abb. 4).

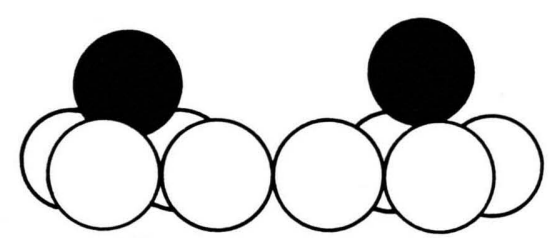

Abb. 4. a- und $a_{1}$-Lage eines Gitterbausteins auf der (110)Fläche.

Berechnungen und Abschätzungen der Bindungs. energien bei arteigener Adsorption liegen von STRANSKI und Suhrmann ${ }^{9}$, Stranski und Eisenloeffer ${ }^{10}$ sowie von MüLLER ${ }^{1}$ vor. Bei diesen Betrachtungen wird angenommen, daß die Bindungskräfte zwischen den einzelnen Bausteinen mit der sechsten Potenz der Entfernung abnehmen und daß sich die Einflüsse der einzelnen Bausteine auf ihre Nachbarn additiv überlagern. Die Bindungsenergie eines Bausteins ergibt sich nach dem KosseL-Schema zu

$$
\varphi_{i}=n_{1} \varphi_{1}+n_{2} \varphi_{2}+n_{3} \varphi_{3}+\ldots,
$$

wobei $n_{1}, \mathrm{n}_{2}, \ldots$ die Anzahl und $\varphi_{1}, \varphi_{2}, \ldots$ die Bindungsenergie der erst-, zweit-... nächsten Nach-

9 I. N. Stranski u. R. Suhrmann, Ann. Phys. Leipzig [6] 1, 153 [1947].

10 I. N. Stranski u. A. Eisenloeffel, Z. Metallk. 41, 10 [1950]. barn bedeuten. Der Übergang von den relativen Bindungsenergien zu den absoluten Bindungsenergien gelingt unter Berücksichtigung der Sublimationsenergie nach Volmer ${ }^{11}$ über die Halbkristallage:

$$
N_{\mathrm{L}} \cdot \varphi_{1 / 2}=\Delta H_{\mathrm{S}}+\frac{1}{2} R T .
$$

Aus der absoluten Bindungsenergie in der Halbkristallage lassen sich die Bindungsenergien in den anderen Lagen leicht berechnen.

Die Oberflächenwanderung kommt dadurch zustande, daß die adsorbierten Bausteine von einer Potentialmulde zur nächsten springen, wenn sie genügend Energie erhalten, um den trennenden Energiesattel zu überwinden. Die Aktivierungsenergie für die Fortbewegung eines Bausteins von einer Lage zur anderen ergibt sich dann aus der Energiedifferenz der Abtrennungsarbeiten aus der anfänglichen Ruhelage und der zu überwindenden Sattellage. Die Aktivierungsenergien für die verschiedenen möglichen Übergänge in den einzelnen Kristallflächen wurden unter der Annahme berechnet, daß der Bindungsanteil der erstnächsten Nachbarn 1,0, der der zweitnächsten Nachbarn 0,45 und der der drittnächsten Nachbarn 0,01 beträgt, während die viertnächsten und die weiter entfernten Nachbarn keinen Einfluß mehr ausüben. Die Aktivierungsenergien der verschiede-

11 M. Volmer, Kinetik der Phasenbildung, Th. Steinkopff-Verlag, Dresden u. Leipzig 1939. 
nen Platzwechselreaktionen sind für Wolfram in der Tab. 2 zusammengefaßt.

Es zeigt sich, daß man sowohl die hier experimentell ermittelte Aktivierungsenergie als auch die bei FEM-Untersuchungen gefundenen Aktivierungsenergien bestimmten Platzwechseln auf den Kristallflächen zuordnen kann. Der nach der radioaktiven

\begin{tabular}{|c|c|c|c|c|c|}
\hline \multirow{2}{*}{$\begin{array}{l}\text { Fläche } \\
h \begin{array}{lll}k & l\end{array}\end{array}$} & \multirow{2}{*}{ Platzwechsel } & \multicolumn{3}{|c|}{ Aktivierungsenergie (kcal) } & \multirow{2}{*}{$\begin{array}{l}\text { Lite- } \\
\text { ratur }\end{array}$} \\
\hline & & $\varphi_{1}$ & $\begin{array}{c}\text { be- } \\
\text { rechnet }\end{array}$ & $\begin{array}{l}\text { experi- } \\
\text { mentell }\end{array}$ & \\
\hline 110 & $\mathrm{a}_{1}-\mathrm{a}-$ Sattel & 0,90 & 32,6 & $\begin{array}{l}30,0 \pm 4 \\
31,6\end{array}$ & 1 \\
\hline & $\mathrm{b}-\mathrm{a}-$ Sattel & 1,80 & 65,4 & 64,4 & 3 \\
\hline & $\mathrm{b}-\mathrm{b}$-Sattel & 1,18 & 42,7 & $\begin{array}{l}41,6 \\
45,3\end{array}$ & $\begin{array}{l}7 \\
5\end{array}$ \\
\hline & $c-b$-Sattel & 2,15 & 78,0 & $80,0 \pm 8$ & 1 \\
\hline 100 & a-a-Sattel & 2,14 & 77,7 & $\begin{array}{l}72,5 \\
74,0\end{array}$ & $\begin{array}{l}4 \\
2\end{array}$ \\
\hline & b-b-Sattel & 2,29 & 83,0 & 85,0 & 1 \\
\hline 111 & a-a-Sattel & 1,94 & 70,3 & 72,5 & 4 \\
\hline $\begin{array}{lll}2 & 1 & 1\end{array}$ & $a-a-S a t t e l$ & 1,49 & 54,4 & $\begin{array}{l}53,2 \\
54,4\end{array}$ & $\begin{array}{l}7 \\
2\end{array}$ \\
\hline
\end{tabular}

Tab. 2. Aktivierungsenergie der einzelnen Platzwechselreaktionen an Wolframoberflächen.

Markierungsmethode ermittelte Wert der Aktivierungsenergie stimmt danach mit dem Wert für den Übergang $a_{1}-a-S a t t e l$ in der (110)-Fläche überein.

Wie ein Vergleich mit den aus den FEM-Untersuchungen erhaltenen Werten zeigt, liegen diese Aktivierungsenergien fast durchweg bei höheren Werten als die hier ermittelte Aktivierungsenergie. Dieses Ergebnis ist durch die unterschiedliche Methodik der Messungen selbst zu erklären. Während bei den Untersuchungen nach der radioaktiven Markierungstechnik stets nur der für den gesamten Diffusionsvorgang geschwindigkeitsbestimmende Schritt erfaßt wird, gelingt bei den FEM-Untersuchungen die gesonderte Beobachtung der Einzelvorgänge, die durchaus höhere Aktivierungsenergien aufweisen können.

Neben Untersuchungen der Oberflächendiffusion auf Wolfram sind hauptsächlich Untersuchungen der Oberflächendiffusion an einer Reihe kubisch-flächenzentrierter Metalle durchgeführt worden. Die Berechnung der Oberflächenplatzwechselenergie nach der Methode von Stranski und Suhrmann, die für kubisch-raumzentrierte Metalle gilt, wurde daher auf kubisch-flächenzentrierte Metalle ausgedehnt. Der größeren Entfernung zweitnächster Nachbarn bei den kubisch-flächenzentrierten Metallen (krz: $r_{2}=1,15 r_{1}$; kfz: $\left.r_{2}=1,41 r_{1}\right)$ wurde dabei dadurch Rechnung getragen, daß der Bindungsanteil der zweitnächsten
Nachbarn nur mit 0,15 berücksichtigt wurde. Die Ergebnisse sind in der Tab. 3 zusammengestellt, in der die berechneten Platzwechselenergien den experimen-
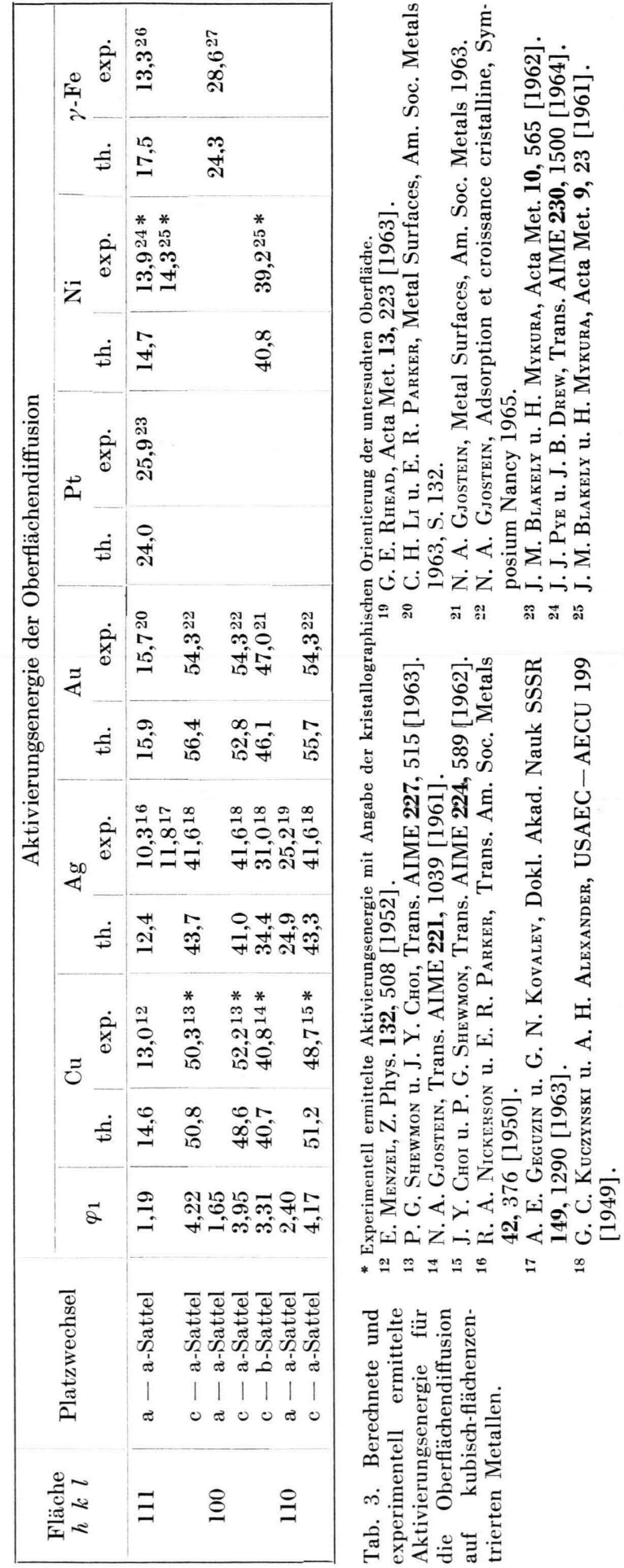
tell ermittelten Aktivierungsenergien gegenübergestellt werden. Es zeigt sich eine gute Übereinstimmung von Theorie und Experiment.

Aus dieser Feststellung folgt, daß die kinetische Methode ein einfaches Verfahren bietet, die Aktivierungsenergie der Oberflächendiffusion von Metallen aus der Verdampfungsenthalpie abzuschätzen.

Zur näherungsweisen Abschätzung der Aktivierungsenergie läßt sich ferner aus dem Vergleich der bekannten Aktivierungsenergien für die Oberflächendiffusion und für die Volumendiffusion eine empirische Regel ableiten, nach der die Aktivierungsenergie der Oberflächendiffusion rund ein Viertel der Aktivierungsenergie der Volumendiffusion beträgt.

$$
\Delta H_{\text {Oberfläche }}=0,25 \Delta H_{\text {Volumen }} .
$$

Die aus den experimentellen Daten ermittelten Werte sind in Tab. 4 zusammengefaßt.

Diese Beziehung hat jedoch, wie betont werden soll, nur empirischen Charakter und eignet sich ledig.

26 I. M. Fedorchenko, Izv. Akad. Nauk SSSR, Otd. Tekhn. Nauk Met. i Toplivo 1952, 560.

27 R. Meclewski, J. Nikliborc u. L. Wojda, Acta Phys. Polon. 21, 189 [1961].

28 K. Hirano, M. Cohen u. D. Duhl, Acta Met. 11, 1 [1963].

29 G. Airoldi u. E. Germagnoli, Energia Nucl. Milan 5, 445 [1958].

80 I. E. Reynolds, B. L. Averbach u. M. Cohen, Acta Met. 5, 29 [1957].

31 G. C. Kuczynski u. G. Cohen, J. Appl. Phys. 21, 1339 [1950].

\begin{tabular}{|c|c|c|c|}
\hline \multirow[b]{2}{*}{ Metall } & \multicolumn{2}{|c|}{ Aktivierungsenergie in keal } & \multirow{2}{*}{$\frac{\Delta H_{\mathrm{ob}}}{\Delta H_{\mathrm{vol}} .}$} \\
\hline & $\begin{array}{l}\text { Oberflächen- } \\
\text { diffusion }\end{array}$ & $\begin{array}{l}\text { Volumen- } \\
\text { diffusion }\end{array}$ & \\
\hline $\mathrm{Cu}$ & $13000^{12}$ & $54000^{31}$ & 0,241 \\
\hline Ag & $10300^{16}$ & $41500^{29}$ & 0,248 \\
\hline $\mathrm{Ni}$ & $14300^{25}$ & $63800^{30}$ & 0,224 \\
\hline $\mathrm{Fe}$ & $13300^{26}$ & $59700^{32}$ & 0,223 \\
\hline $\mathrm{Zn}$ & $6200^{36}$ & $25400^{33}$ & 0,244 \\
\hline $\mathrm{W}$ & 31600 & $120500^{34}$ & 0,262 \\
\hline $\mathrm{Au}$ & $15700^{20}$ & 4210028 & 0,373 \\
\hline $\mathrm{Pt}$ & $25900^{23}$ & $68700^{35}$ & 0,387 \\
\hline
\end{tabular}

Tab. 4. Vergleich der Aktivierungsenergien für Oberflächendifiusion und Volumendiffusion.

lich zur größenordnungsmäßigen Abschätzung der Aktivierungsenergie der Oberflächendiffusion.

Eine recht genaue Abschätzung der Aktivierungsenergie der Oberflächendiffusion aus der Verdampfungsenthalpie ermöglicht dagegen das kinetische Verfahren der Betrachtung der einzelnen Bindungsenergien.

Für die Unterstützung dieser Untersuchungen danken wir der Deutschen Forschungsgemeinschaft.

32 F. S. Buffington, I. D. Bakelar u. M. Cohen, Phys. Powder Metallurgy 1951, 404.

33 H. B. Huntington, G. A. Shirn u. E. S. Wajda, Phys. Rev. 87, 211 [1952].

34 W. Danneberg, Metall 15, 977 [1961].

35 G. V. Kidson u. R. Ross, Radioisotopes Sci. Res., Proc. Intern. Conf. Paris 1957, Pergamon Press Ltd., London 1958, Vol. 1, p. 185.

36 G. Klaffke, Dissertation, Techn. Universität Berlin 1958. 